\title{
Brown-Séquard syndrome due to Semple antirabies vaccine: case report
}

\author{
H Sathyanarayana Swamy MD DM, A Vasanth DM, Sasikumar MBBS, DM \\ Department of Neurology, National Institute of Mental Health and Neuro Sciences, \\ Bangalore - 560029, India.
}

We report a patient with a Brown-Séquard syndrome due to Semple type antirabies vaccine, with subclinical electrophysiological abnormalities, who recovered with immunosuppressants.

Key words: Brown-Séquard sydrome; antirabies vaccine; immunosuppressants; rabies; vaccination.

\section{Introduction}

Although neurological complications of the Semple antirabies vaccine are common ${ }^{1-7}$, development of the Brown-Séquard syndrome has not previously been reported. We describe a patient with signs and symptoms resembling the Brown-Séquard syndrome following Semple antirabies vaccine and his complete recovery with immunosuppressive therapy.

\section{Case report}

A 28 year old man was admitted with neurological complications due to the Semple type of antirabies vaccine (ARV). He was bitten by a healthy dog one month before admission. The patient received 4 doses of vaccine on 4 consecutive days beginning on the day following the dog bite. One month after the first dose of ARV he developed pain around the thorax for 2 days. On the third day he had urinary retention, and a day later developed weakness of the right lower limb and inability to appreciate touch and pain sensation in the left half of the body below the middle part of the chest.

On examination, his higher mental function, cranial nerves and upper limbs were normal. There were pyramidal signs in right lower limb with $0-3 / 5$ (MRC) weakness, spasticity, brisk deep tendon reflexes and an extensor plantar response. Pain, touch and temperature sensations were impaired over the trunk below T5 on left side and in left lower limb. Joint sensation was normal bilaterally. Other systems were normal.

\section{Investigations}

Haematological indices were normal as were estimation of blood sugar, urea, sodium, potassium and the liver function tests. Lumbar cerebrospinal fluid analysis, $x$-ray of the thoracic spine and spinal computerised tomography were also normal, as were the electroencephalogram, visual evoked potentials and brain stem auditory evoked responses. The somato sensory evoked potential of the posterior tibial nerve (N 39) was $39.5 \mathrm{~m} / \mathrm{sec}$ bilaterally and the wave form was poorly defined. Electromyography was done with concentric needle electrode on 4 channel DISA 1500 model. The right abductor pollicis brevis, right quadriceps and right tibials anterior were sampled. Electromyography of the right abductor pollicis brevis was within normal limits. There was no spontaneous activity in the right quadriceps and the right tibialis anterior and the motor unit potentials were of normal amplitude and duration, but the firing rate was reduced to $4 /$ second. Recruitment was incomplete in both these muscles, suggesting probably upper motor neuron lesion. Nerve conduction parameters are shown in Table I. In summary, abnormalities of nerve conduction were bilateral absence of $F$ response from common peroneal nerve and absence of Hoffman's reflex from the left posterior tibial nerve suggesting radicular involvement. There was decreased amplitude of the left sural sensory action potential as compared to the right, with normal conduction velocity suggesting a mild postganglionic axon loss neuropathy. 
Table I Nerve conduction parameters

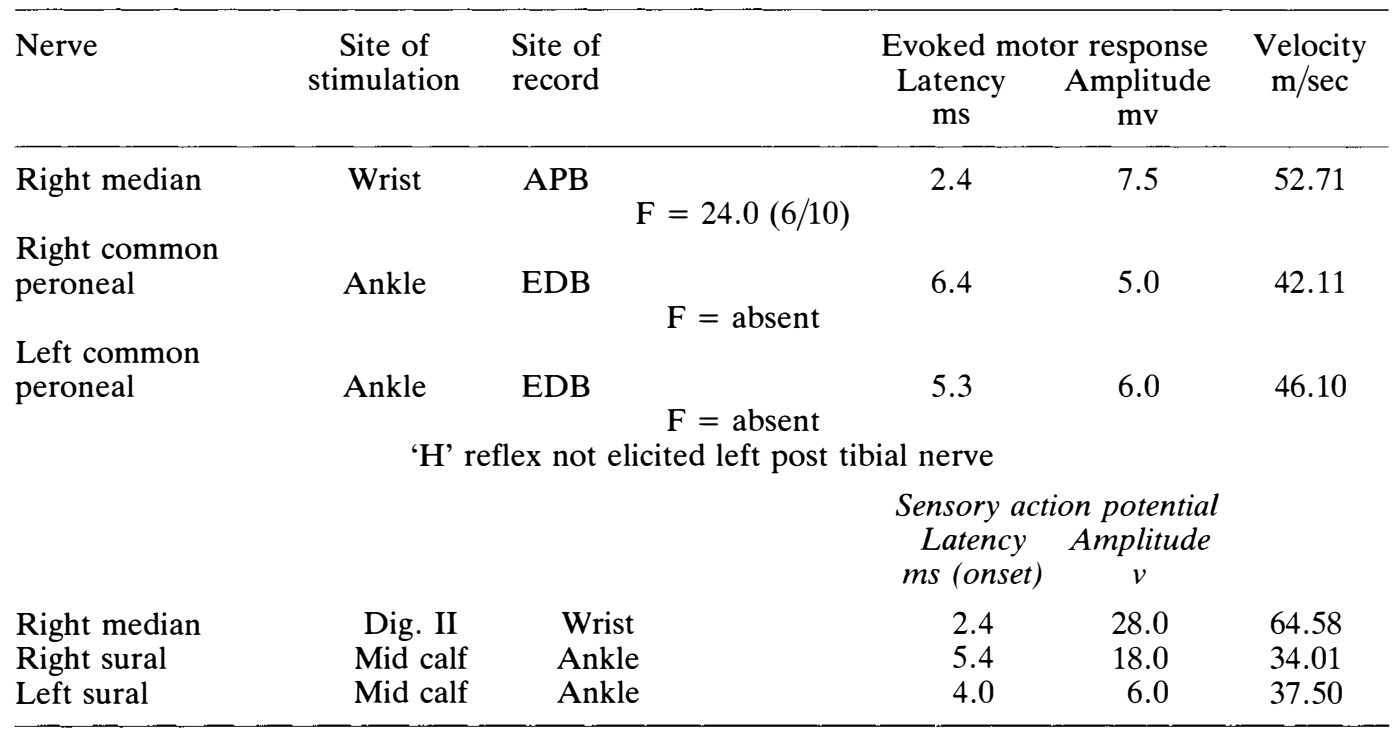

\section{Treatment response}

Injections of dexamethasone $16 \mathrm{mg}$ daily were given from one month after the onset of illness for 15 days and tapered off over one month. Intravenous injections of cyclophosphamide $200 \mathrm{mg}$ daily were given for 10 days from the same date. The patient gradually improved and was free from neurological deficits at the last follow up, 4 weeks later.

\section{Discussion}

Semple vaccine, a suspension of phenol inactivated rabies virus in the brain of sheep or higher mammals, is used in many developing countries. The neuroparalytic accidents due to this vaccine occur in about 1 in 220 cases and the failure rate is about $11 \%{ }^{8,9}$ Suckling mice brain (SMB) vaccine containing inactivated rabies virus is used in Latin America and is associated with neuroparalytic accidents of $1 / 7865 .^{2}$

Duck embryo vaccine (DEV), a non neural vaccine, was introduced during 1959. Label and Batts ${ }^{3}$ reviewed literature in 1982 and found 24 cases of neurological compli- cations due to this vaccine.

Human diploid cell (HDC) a non neural rabies vaccine was introduced during 1976. The neurological complications due to this vaccine are rare and it is highly immunogenic in contrast to Semple vaccine. ${ }^{9}$

The neurological complications are immunologically mediated and immunosuppressants are of great value as in the present case. . $^{1,10-13}$

With the present knowledge, hemisection of the spinal cord experimentally should produce loss of pain and thermal sensations on the opposite side of body and loss of proprioceptive sensation with pyramidal signs of the same side of lesion. However this is rarely seen clinically in complete form, partly because of the anatomy of blood supply, and in this case posterior column sensation was preserved on the side of the lesion.

The time has come to use human diploid cell vaccine exclusively even although stores of Semple vaccine may still be available and be much cheaper. The complication rate of Semple vaccine is so high that it hardly seems justifiable to use it nowadays. 


\section{References}

1 Sathyanarayana Swamy H, Shankar SK, Chandra PS, Aroor SR, Shivaramakrishna A, Kaliaperumal VG (1985) Neurological complications due to beta-propiolactone (BPL) inactivated antirabies vaccination. $J$ Neurol Sci 63: 111-128.

2 Toro G, Vergara I and Roman G (1977) Neuroparalytic accidents of antirabies vaccination with SMB vaccine. Arch Neurol 34: 694-700.

3 Label LS, Batts DH (1982) Transverse myelitis caused by duck embryo rabies vaccine. Arch Neurol 39: 426-430.

4 Helm EB, Dillmann W, Stille W (1978) Experience with new vaccines in rabies vaccination treatment. Munch Med Wschr 120: 293-296.

5 Boe E, Nyland H (1980) Guillain-Barre syndrome after vaccination with human diploid cell rabies vaccine. Scand J Infect Dis 12: 231-232.

6 Uchimura I, Shiraki H (1957) A contribution to the classification and the pathogenesis of demyelinating encephalomyelitis. J Neuropath Exp Neurol 16: 139-208.

7 Appelbaum E, Greenberg M, Nelson J. (1953). Neurological complications following anti-rabies vaccination. JAMA 151: 188-191.

8 Hemachudha T, Phanuphak P, Johnson RT, Ratanavongsiri J, Siriprasomsup W (1987) Neurologic complications of Semple-type rabies vaccine: clinical and immunologic studies. Neurology (Minneapolis) 37: 550-556.

9 Warrell MJ, Suntharasamai P, Sinhaseni A, Phanfung R, Falquet JCV, Bunnag D et al (1983) An economical regimen of human diploid cell strain anti-rabies vaccine for post-exposure prophylaxis. Lancet II August 6th: 301-303.

10 Brij Kishore, Meharotra MP, Hazra DK, Wahal PK, Bansal OP, Patney NL and Agrawal YB (1978) A study of circulating anti-bodies in cases receiving antirabic inoculation with reference to the neurological complications. J Ass Phys India 28: 479-484.

11 Briggs GW and Brown MW (1960) Neurological complications of antirabies vaccine-treatment with corticosteroids. J Am Med Assoc 173: 802-804.

12 Ferraro A and Roizin L (1953) Experimental allergic encephalomyelitis during and following cortisone acetate treatment. J Neuropath Exp Neurol 12: 373-386.

13 Garrison SC (1952) Encephalomyelitis complicating antirabies vaccination treated with cortisone. Am J Med 12: 135. 